\title{
MICHAEL TOXITES Y LOS VOCABULARIOS PLURILINGÜES DE ONOMASTICA (1574)
}

\author{
MICHAEL TOXITES AND THE MULTILINGUAL \\ VOCABULARIES IN ONOMASTICA (1574)
}

\author{
Luis Pablo NúÑez \\ Universidad de Granada \\ luispablo@ugr.es
}

RESUMEN: Este artículo analiza los vocabularios contenidos en Onomastica (1574) de Michael Toxites (seudónimo de Michael Schütz), una obra no estudiada aún en el ámbito hispánico. Semejante a obras posteriores de sistematización terminológica, como las de Caspar Bauhin o Christian Mentzel para el ámbito botánico, Onomastica recopila voces de sustancias minerales y vegetales usadas en la realización de compuestos alquímicos durante el Renacimiento. Dividida en dos partes, una primera temática y una segunda alfabética, la obra recoge voces comunes y técnicas en varias lenguas (latín, hebreo, árabe, alemán, francés, español, italiano e inglés). Este estudio realiza una aproximación global al autor y la obra, describe la estructura de los dos repertorios que la componen, analiza algunos de los términos que incluye debido a su temprana datación y, por último, establece su relación con otras obras paracelsísticas similares de su época.

Palabras clave: Michael Toxites (1515-1581); Onomastica; Paracelso (1493-1541); vocabulario plurilingüe; lexicografía de especialidad; terminología.

Abstract: This article analyzes the two vocabularies contained in Onomastica (1574), a work by Michael Toxites (pseudonym of Michael Schütz) not yet considered in Hispanic lexical studies. Similar to botanical works by Caspar Bauhin or Christian Mentzel, Toxites' Onomastica compiles terms related to mineral and vegetal substances used in Renaissance alchemic and chemical compounds. Divided in two parts, the first ordered by subject, the second alphabetically, the work list common and technical terms in several languages (Latin, Hebrew, Arabic, German, French, Spanish, Italian, and English). This study explains the structure of both glossaries, it describes some of the terms included, emphasazing its importance for Spanish language in view of its early datation, and, finally, it tries to establish some of its links to other similar Paracelsian works.

Keywords: Michael Toxites (1515-1581); Onomastica; Paracelsus (14931541); multilingual vocabulary; specialised lexicography; terminology.

Recepción: 30 de septiembre de 2017; aceptación: 24 de abril de 2018. 
Propósito de este estudio

Los siglos XVI y XVII fueron de gran desarrollo en el ámbito de las disciplinas científicas. Tras la recuperación de textos de los autores clásicos y su difusión mediante la imprenta, se realizaron comentarios que validaron o corrigieron lo indicado en ellos. Los contactos por carta y envíos de materiales entre los estudiosos promovieron también la divulgación de nuevas teorías por toda Europa. Surgieron métodos experimentales que, mediante la observación directa de la naturaleza y la manipulación de sustancias, permitieron el desarrollo empírico de varias de las ciencias actuales.

La botánica, por ejemplo, unida previamente al estudio del reino animal y mineral como parte de la filosofía natural, y concebida como una rama auxiliar de la medicina, fue ampliando su función subsidiaria de composición de remedios medicinales y, tras los continuos esfuerzos de descripción y clasificación de las plantas, se convirtió en una disciplina independiente. Del mismo modo ocurrió con la química: los pasos dados durante el Renacimiento y Barroco la consolidaron como ciencia en el siglo XVIII.

El desarrollo de la química está ligado a la medicina y a la farmacia, por lo que se refiere a la preparación de medicamentos, y sus orígenes, a las experimentaciones con sustancias de la alquimia. Ésta surgió como un modo de transformación de metales innobles en nobles (oro y plata) y en la búsqueda de la longevidad o, incluso, la inmortalidad, pero acabó convirtiéndose en un sistema filosófico misticista. Con la llegada del Renacimiento, se rechazó esta perspectiva mágica, se puso en entredicho el galenismo y, con obras como las de Paracelso (Philipp Theophrast von Hohenheim, 1493-1541), surgió una nueva concepción ${ }^{1}$, según la cual, dicho de forma muy sintetizada, el

${ }^{1}$ La bibliografía sobre la obra de Paracelso y sus seguidores es muy abundante y fue realizada en buena medida a finales del siglo xIX: SUDHOFF 1894, luego ampliado en SudHoff 1958 (Onomastica se cita en núm. 154). Asimismo, FERGUSON (1877-1896) incluye varias partes, entre las cuales la quinta, An alphabetical catalogue of the different editions of the works of Paracelsus (1893), ha sido reeditada en facsímil: Mansfield Centre, CT, Martino Publishing, 2003. Para un análisis amplio del complejo desarrollo del paracelsismo y la recepción de la obra de Paracelso durante los siglos XVI y XVII, que no podemos realizar aquí, remitimos a los tres volúmenes del Corpus Paracelsisticum publicados por DeGruyter, respectivamente, en 2001, 2004 y 2013. 
hombre debía su salud a la conexión con la naturaleza, y para restablecer los desequilibrios originados por las enfermedades, podían hallarse remedios mediante sustancias producidas por la misma naturaleza combinadas químicamente (pues "en transmuant les choses..., la préparation donne aux choses ce que la Nature n'a pu, à savoir la maturation, et la science du Médecin est de maturer", Sarcilly 1950 [1631], p. 22). Esta concepción abrió a la alquimia las puertas a la experimentación más allá de la filosofía misticista con compuestos minerales, destilaciones, bálsamos y preparados de antimonio y mercurio (como ejemplifican los Quatre livres des secrets de la Medecine et de la Philosophie chymique de Jean Liebaut, 1573) ${ }^{2}$. Con los avances posteriores de la medicina y de la química, la alquimia fue perdiendo su razón de ser hasta que se la redujo a disciplina esotérica de ámbito extraacadémico, como ocurre hoy con la astrología. Durante el siglo XVII, sin embargo, la química moderna no había aún aparecido y la alquimia estaba en su fase más fecunda de experimentación.

Teniendo, pues, en cuenta el contexto anterior, el propósito de este estudio es describir Onomastica (1574) de Michael Toxites, uno de los principales comentadores de Paracelso. La obra no es desconocida, pero sólo se ha tratado desde la perspectiva de la historia del paracelsismo; no se ha estudiado nunca, que sepamos, en el ámbito hispánico ni se ha visto su importancia para trazar la historia de ciertas voces del español. La perspectiva que se adoptará será por ello filológica y, más concretamente, lexicológica, pues la obra está compuesta por dos vocabularios. Nuestro objetivo será mostrar que es una obra relevante para la difusión y fijación de algunos términos de especialidad de la lengua española, ya que por su fecha de publicación, 1574, documenta voces que no habían aparecido en repertorios léxicos anteriores o se encuentran entre sus dataciones más tempranas.

Primeramente, haremos una semblanza biográfica del autor, no conocido en el ámbito hispánico. Luego, describiremos la estructura de la obra, analizaremos sus términos, determinare-

${ }^{2}$ Sobre la divulgación del paracelsismo en las regiones alemanas y la censura que sufrió en ciertas regiones de Europa, puede verse RoDRíguEz Guerrero 2001. Sobre la reunión de los escritos de Paracelso, la recepción de su doctrina y el papel de las traducciones de sus obras al latín o los comentarios realizados por Toxites y Adam von Bodenstein, pueden verse los estudios de Daniel 2007, pp. 199-200, y Patin 2010, pp. 206-208. 
mos con ejemplos cuáles fueron dos de sus fuentes y, finalmente, mostraremos su relación con otras obras de su época, que, como la de Toxites, buscaron una sistematización terminológica que permitiera con su consulta el avance de las disciplinas.

\section{VIDA Y OBRA DE TOXITES}

Michael Toxites, llamado realmente Johann Michael Schütz, nació en Sterzing (actual Tirol italiano, al sur de Innsbruck) el 19 de julio de $1514^{3}$. Su vida giró en torno al triángulo Basilea-Estrasburgo-Tubinga. Sus primeros estudios los realizó en Dillingen (actual Alemania); en 1532 obtuvo el Baccalaureus Artium (bachillerato en artes) en Tubinga. Tras algunos estudios en la Universidad de Pavía (1535), obtuvo el título de Magister en 1542 en Wittenberg. Fue profesor de latín en Bad Urach (Baden-Württemberg) desde 1537, pero allí se le acusó de haber escrito un libelo contra el duque de Württemberg y se le expulsó de la ciudad. Se trasladó a Basilea, donde siguió ejerciendo, y luego a Estrasburgo (1542-1545), pero al poco regresó de nuevo a Basilea (1546 y 1548-1549). Tras realizar estudios de medicina en Estrasburgo entre 1551 y 1556 (se graduaría en 1562) volvió a Tubinga, donde fue rehabilitado de su destierro y nombrado profesor de retórica y poética (15561560). En 1564 ya ejercía como médico en Estrasburgo y, tras entrar en contacto con personas como Gerhard Dorn, Adam von Bodenstein y Johann Huser, se consagró al estudio de la obra de Paracelso y a la experimentación en laboratorio con sustancias químicas. En 1574 se estableció en Haguenau (Alsacia), donde murió en 1581.

Por su formación como profesor de latín y retórica publicó numerosas obras, varias de ellas comentarios a autores grecolatinos, como los Commentarius Michaeli Toxitae, Rhaeti poetae laureati in orationem M. Tulij Ciceronis pro C. Plancio (1551), Com-

${ }^{3}$ La fuente principal para la biografía de Toxites sigue siendo el libro de Schmidt (1888). Una versión reducida se puede consultar en el Dictionnaire historique de la Suisse, http:/ / www.hls-dhs-dss.ch/textes/d/D42851.php. Asimismo, cf. Soukup und MAYER 1997 ("9.3. Zur biographie des Michael Toxites”, pp. 236-242) y Soukup 2007. En este apartado realizamos sólo una breve aproximación a su vida y obra para contextualizar la que nos interesa analizar, Onomastica; quien desee ahondar en la labor y la figura de Toxites, en general, puede acudir a los estudios citados, más amplios y exhaustivos. 
mentarius Micaeli Toxitae Rhaeti poetae laureati in Philippicam M.T. Ciceronis alteram (1552), Commentarii Micaeli Toxitae... in libros quatuor Rhetoricorum ad C. Herennium (1556), Scholia Micaeli Toxitae... in Theocriti Idyllion primum (s.a.), etc. También publicó una pequeña obra didáctica para la educación de príncipes titulada

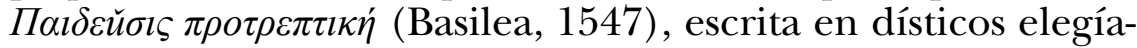
$\cos ^{4}$. Sus primeras obras fueron sin embargo poemas en latín (de hecho, en 1544 había recibido la distinción imperial de poeta laureatus): Querela anseris, vel de Ingratitudine hominum elegia, publicada en Estrasburgo en 1540, seguida de otras como las Sententiae Nili episcopi et martyris (1543), o las elegías Manes clariss. Virorvm D. Wolfgangi F. Capitonis, Simonis Grynaei, et Iacobi Bedrotti (1542), Epicedion... lavreati, in funere clariss. . viri domini Jacobi Sturmii (1554), etcétera ${ }^{5}$.

Durante los últimos años de su vida, ya como médico integrante de los círculos cultos de su época -en unos poemas de 1567 contra otro médico se declaraba ya seguidor de Paracelso (Toxites 1567)-, prologó o editó numerosas obras, algunas de ellas relacionadas con la alquimia y con Paracelso, como Archidoxa (Estrasburgo, 1570), Astronomia Magna (1571), Zwey Tractatus des hocherfarnen und bewaertisten teuetschen Philosophi (1572), los tratados De lapide philosophorum (1572), De vita longa (Fünff Bücher vom dem langen Leben, Estrasburgo, 1574), Libri XIV paragraphorum (1575) o Aureoli Theophrasti Paracelsi eremitae, philosophi summi Operum latine redditorum tomus I (1575, en que fue uno de los traductores), en colaboración con estudiosos como Gerard Dorn y Adam von Bodenstein (para la labor en cada una de ellas puede consultarse el Corpus Paracelsisticum citado).

No sólo publicó las obras de Paracelso, sino también las de otros autores, como la de Raimundo Lulio, Libelli aliquot chemi-

${ }^{4}$ Puede consultarse un ejemplar digitalizado en la Bayerische StaatsBibliothek Digital, http: / / www.mdz-nbn-resolving. de / urn/ resolver. pl?urn= urn: nbn: de:bvb:12-bsb10908176-7, como también, para sus otras obras, diferentes bibliotecas virtuales, la de la Universidad Complutense, por ejemplo. Como punto de partida, puede verse en el Universal Short Title Catalogue (USTC, http:// ustc.ac.uk/) la ficha del autor y los ejemplares de cada título localizados en bibliotecas públicas.

${ }^{5}$ Más adelante, algunas obras lo presentan como Michaeli Toxitae Rhaeti, poetae $\mathcal{E}$ Comitis Palatini Caesarei, E̋ medici Argentoratensis (1564); en otras se lo vincula con la vida académica de Tubinga: Oratione publice in celeberrima Tubingensi Academia a professoribus pulicis per hoc trimestre habitae [Theodoricus Schnepfius; Matthias Garbitius Illiricus; Michael Toxites; Ioannes Secceruitius], Tubingæ, Morhardus, 1557. 
ci: nunc primum, excepto Vade mecum in lucem opera doctoris Toxite editi (Basilea, 1572 y Colonia, 1573; Basilea, 1577, 1600, etc.), en que se trataba la transmutación de los metales; Introductio in divinam chemicae artem del médico de Ferrara Pietro Antonio Boni (Basilea, 1572) o la Filosofía hermética de Bernard le Trévisan (Von der hermetischenn Philosophia, das ist, vom gebenedeiten Stain der Weisen, Estrasburgo, 1582).

Tras su muerte, algunos de sus abundantes escritos siguieron reimprimiéndose o incluyéndose en colectáneas hasta treinta años más tarde. Onomastica (1574), que recoge en una de sus partes un léxico de las voces presentes en las obras de Paracelso, pertenece a ese período final, tan prolífico, de su vida.

De Toxites se conserva alguna carta manuscrita en la Waller Manuscript Collection de la Universidad de Uppsala (colección de autógrafos relativos a la historia de la medicina y de la ciencia, con especial atención a las redes que se establecieron entre científicos de todas las épocas, desde la Edad Media al siglo Xx, Waller ms. ch-00479) ${ }^{6}$.

\section{ONOMASTICA. DESCRIPGIÓN DE LA OBRA}

Desde el punto de vista bibliográfico, Onomastica es una obra voluminosa de pequeño formato dirigida a médicos, boticarios y estudiosos de la obra de Teofrasto. Su título completo es el siguiente: Onomastica II. I Philosophicum, medicum, synonymum ex varijs vulgaribusque linguis. II Theophrasti Paracelsi: hoc est, earum vocum, quarum in scriptis eius solet usus esse, explicatio. Nunc primum in commodum omnium philosophiae, ac medicinae Theophrasticae studiosorum, cuiuscunque nationis sint: fideliter publicata. $=$ Gründliche Erklärung in allerlei Sprachen der philosophischen, Medicischen vnd Chimicischen Namen, welcher sich die Arzet, Apoteker, auch Theophrastus zugebrauchen pflegen. Nun erstmals idermäniglichen zu meherem nuz/ richtigerem Verstand und förderlicher lesung der Theophrastischen und sonst Bucher/ ordentlich und fleisig inn truck gefärtiget ${ }^{7}$. Argentorati [= Estrasburgo], Per Bernhardum Iobinum, 1574. [16],

6 The Waller Manuscript Collection, Uppsala University Library, http:/ / waller.ub.uu.se/24434.html.

7 El texto alemán va en gótica, frente al texto latino, en redonda. La ortografía de algunas voces ha cambiado hoy día: Arzet $>$ Arzt; Apoteker $>$ Apotheker, etcétera. 
382, [2], 383-490, [4] p. ; 8. ${ }^{\circ}(16 \mathrm{~cm})$. Cada parte (Onomastica I, Onomastica II) lleva un colofón propio, pero idéntico, que transcribimos: Argentorati. Per Bernhardum Iobinum. Anno Salutis Humanae M.D.LXXIIII.

Teniendo en cuenta la buena conservación general de las obras botánicas y médicas, la de Toxites no tuvo demasiada difusión: de los en torno a cuarenta ejemplares que localizo en bibliotecas públicas de todo el mundo, no hay ninguno en España. Sí hay ejemplares en la Biblioteca Nacional de Francia y en la Academia de Medicina de París, en la Bodleian, la Biblioteca Nacional de Austria y en la Wellcome Library de Londres, así como en la Biblioteca Nacional de Medicina de Bethesda (Estados Unidos), en la Academia de Medicina de Nueva York, en la Universitaria de Boston y Washington y en Yale, pero el grueso de sus ejemplares se conserva en Alemania: Augsburgo, Berlín, Dresde, Hamburgo, Jena, Múnich, Stuttgart, Tubinga y Wölfenbuttel, entre otras bibliotecas ${ }^{8}$. Para este trabajo hemos manejado el ejemplar de la Biblioteca Nacional de Francia 8-T27-5 y la copia digitalizada de la Biblioteca Estatal de Múnich, Bibl.Sud. 1463 (VD16 T 1769).

Desde el punto de vista de su estructura, la obra se divide en dos partes, tal y como indica el título: la primera es un léxico plurilingüe que recoge las voces de las sustancias usadas como simples (remedios medicinales ${ }^{9}$ ), muchas de las cuales proceden de la cabalística y la química ("non pauca à Chemistis ficta usurpavit", señala en a3r); la segunda es un vocabulario alfabético de las voces aparecidas en los libros de Paracelso, en parte recogidas por él al editar sus obras y en parte por otros, como él mismo menciona:

adiecque aliud Theophrasticum: hoc est, earum vocum, quibus Theophrastus in suis scriptis usus est, explicationem: partim ab aliis, partim ab me ipso ex eius libris congesta, in ordinem alphabetici redegi (Præfatio, a3v).

${ }^{8}$ La relación completa de estos ejemplares con sus signaturas se da en Pablo NúÑez 2012, pp. 171-172. USTC (http:// ustc.ac.uk/ index.php/ record/78303) señala una docena de ellos.

9 “«Simples», es decir, las sustancias de procedencia vegetal, mineral o animal, con aplicación terapéutica, que se pueden utilizar bien sea de forma aislada o bien mezclándose entre sí para formar los compuestos”, como indica Gutiérrez Rodilla 2007, p. 50. 
Se trata, pues, de dos repertorios léxicos, uno complementario del otro, como muestra alguna que otra remisión del segundo vocabulario al primero: morelum, solatrum, Nachtschatten/Vide primo Lexico in herbis, etcétera ${ }^{10}$.

Este primero es una nomenclatura organizada temáticamente (aunque luego veremos las particularidades de esta ordenación) con lengua de entrada en alemán y equivalencias en latín, griego, francés, español, italiano y, ocasionalmente, inglés, árabe y hebreo ${ }^{11}$. El segundo, Onomasticon alterum Theophrasti Paracelsi: hoc est, earum uocum, quibus Theophrastus Paracelsus in suis scriptis usus est, per varias linguas explicatio, es en realidad una obra alfabetizada por la voz latina, seguida de su definición/ explicación, más la equivalencia/ explicación en alemán y francés, con ocasional inclusión de español e italiano. En esta segunda parte, el inglés aparece muy raramente (aunque hay algunos casos, poquísimos, como en "Abefsi..., Gall. La derniere matiere demourant des viandes. Hisp. demorante de ceuo. Angl. materie of the meate; o Assaliæ, vermes... Gall. ver de bois. Hisp. tarma. Angl. à Tymber Vuorme").

Si el primer vocabulario indicaba la varietas de un término, las variaciones terminológicas según el uso en diferentes autores o regiones, en el segundo se dan las explicaciones de los términos, esto es, las definiciones. El primero es más amplio porque incluye nombres de plantas y animales; el segundo es más reducido porque omite los nombres de plantas comunes: "Herbarum communia nomina, quae in herbariijs reperiuntur, omisi: rariora posui" (a4v). La obra en su conjunto carece de

10 También en la voz litargirio: "Lithargirium notum est: Theophrastus autem sic etiam vocat spumam Mercurij; alias argenti spuma lithargyros. Silberschaum/Glet. Gall. escume d'argento. Hisp. litargirio. Vide supra p. 20 D" (el subrayado es nuestro; remite al primer vocabulario, Onomastica I, en que, por cierto, no se daba litargirio como equivalente hisp., sino almartaga). En el apartado "Análisis de algunas voces castellanas" estudiaremos con detalle esta voz.

11 Transcribimos más adelante el párrafo correspondiente. El prólogo indica una ordenación alfabética: "Tractaturque per literas Alphabeti priores, ut commodius postea in Indice quæuis materia quæri possit" (f. A1r), pero peculiar, como veremos. Aunque normalmente se usan caracteres griegos para los términos en esta lengua, ocasionalmente también se utiliza tipografía redonda y la marca "Græc." para identificarlos. En el caso del árabe o hebreo, sólo se usa el alfabeto latino y, posteriormente, la marca "Arab." / "Hebr.": "atramentum griseum, terra nigra: zez nigrum: Arab." 
ilustraciones, lo que refuerza más la voluntad lexicológica con que fue escrita.

A los léxicos preceden unos preliminares que proporcionan algunos datos relevantes. La obra está dedicada a los hermanos Maximilian y Viktor August Fugger; el primero, caballero de la orden teutónica, y el segundo, praeposito de Ratisbona y canónigo de Passau, de las regiones de Kirchberg y Weissenhorn (cerca de Ulm $)^{12}$. El prefacio, escrito por Toxites, está fechado a mediados de marzo de 1574 y firmado en Haguenau, localidad cercana a Estrasburgo, entonces de ámbito germano.

Le sigue una nota al lector escrita en latín por Johann Fischart $^{13}$ donde se indica que fue el encargado de la edición de la obra, para la cual completó y ordenó el primer vocabulario bajo petición del mismo Toxites, lo que está en consonancia con lo afirmado por otros investigadores que sugieren que, como al final de cada uno de los dos repertorios hay una hoja con el colofón del impresor, cada una de las partes que componen la obra podría ser independiente de la otra: el primer vocabulario pudo haber sido realizado principalmente por Toxites, y el segundo, por Fischart ${ }^{14}$.

El prólogo es además relevante porque, más allá de defender la importancia de conocer la obra y el léxico de Paracelso, señala la relación de Fischart con Toxites, muestra una concepción filológica de la obra al referirse a ella como un léxico y nombra algunas de las fuentes -que veremos luego- que se utilizaron para componer los vocabularios.

12 Sobre Viktor August Fugger y Toxites, véase Soukoup und Mayer 1997, caps. 1.4 (pp. 16-22) y 9 (pp. 224-242).

13 Johann Baptist Fischart (Estrasburgo, 1546-Forbach, 1590) fue un estudioso de la literatura, poeta y abogado en la corte de Espira, graduado en Basilea, pero ha pasado a la posteridad como traductor al alemán de la obra de Rabelais y como uno de los mayores agitadores alemanes durante la Contrarreforma. Defensor de las ideas protestantes, publicó sátiras morales contra la Iglesia católica romana y sus órdenes religiosas (franciscanos, jesuitas) en las que hacía ingeniosos juegos de palabras: Die Wunderlicht legend (1580), contra los jesuitas, y Antihispanus, das ist Widerlegung spanischer unart, Angemaßter der Kron Frankreich unzeitigen beherrschung... aus dem Frantzösischen Antiespagnol verdolmetschet (1590), traducción de L'Anti-Espagnol del abogado francés Antoine Arnauld (1560-1619). Muchas de sus obras fueron publicadas por el parentesco que tenía con uno de los impresores de Estrasburgo, Bernhard Jobin, casado con su hermana, y quizá haya sido éste el vínculo por el que pudo conocer a Toxites y encargarse de parte de su obra. Cf. SOMMERHALDER 2018.

14 Así se indica en Tonelli 2006, p. 122. 
Allí también se señala que si en todas las disciplinas es provechoso conocer varias lenguas, más aún lo es conocerlas en la Medicina, para poder ejercerla con las hierbas y los minerales necesarios. Ya lo hizo así Dioscórides -señala- cuando obtuvo los diferentes nombres de las plantas de los distintos pueblos: romanos, árabes, sirios, hebreos, griegos, caldeos, egipcios, chipriotas, etruscos, latinos, etc. Nombra también a Valerius Cordus, uno de los comentaristas del Dioscórides, y a Conrad Gesner, autoridad ineludible, pues fue una figura clave de la historia natural del Renacimiento, autor de un Catalogum plantarum, también en varias lenguas (latín, griego, alemán y francés), que "por muy imperfecto que fuera" -escribe- mostró muchos avances en la investigación de las plantas. Cita también los trabajos de Amato Lusitano, Valerius Cordus, Mattioli, Ruelio, Lonizer y Hadrianus Junius ${ }^{15}$ que, como veremos, fueron las fuentes de este trabajo, resultado de una labor de recopilación. Véase el siguiente fragmento en que se indica todo lo que hemos señalado:

Hunc imitando postea D. Conradus Gesnerus (ornamentum Germaniæ) Catalogum plantarum Latinè, Græcè, Germanicè, \& Francogallicè hoc seculo conscripsit, qui quamuis multum imperfectus sit, ut ipsemet in Nuncupatoria fatetur, \& experientia ipsa hodie comprobet, quòd interim ab eius obitu multa in herbarum inuestigatione correcta, innouata, inuentaque reperiantur: attamen eo labore iudicium suum (quod permagni habendum) de huius modi futuro Lexico, quale hoc nostrum est, tacite innuit: idque etiam commendationis uicepræmissum gaudemus. Taceo Amati Lusitani, Andreae Mathioli, Ioannis Ruellij, Ioh. Loniceri, Hadriani Iunij, aliorumque doctissimorum uirorum in eodem genere tractionis utilissimos labores, his nostris in boni ominis præiudicium (ut ita loquar) ac comprobationem uergentes.

Quamobre speramus, neminem sani iudicij hominem hanc operam nostram, quam cohortatione clarissimi uiri D. Doctoris Michaelis Toxitæ in declarando, adaugendo \& uulgaribus linguis huic composito prius Onomastico addendo, suscept[i]mus, improbaturum esse: imò ipsi Doctori magnas gratias habiturum, quod opus hoc publicandi author extiterit (Fischart, Prólogo al lector).

Pero esta labor de recopilación tiene una particularidad: frente a las obras citadas que la precedieron, los vocabularios de

${ }^{15}$ Para la descripción de estas obras, véase PABLo NúÑEz 2010 y 2012. 
Onomastica son un "índice", un léxico dirigido a médicos, boticarios y estudiosos de la medicina que necesitan conocer los diversos nombres de las plantas, como el mismo Fischart señala, en que la prioridad son las lenguas vulgares, no las lenguas cultas, aunque también se consideren y sea el latín la lengua eje empleada como lema (por ser la lengua culta en Europa, común a autores de diferentes procedencias, y por ser la más utilizada en obras técnicas impresas). Y, dentro de las lenguas vulgares, los varios sinónimos con que son conocidas ciertas plantas o minerales se colocan en primer lugar, antes de las lenguas clásicas:

Collocauimus primo loco nomina materiarum Germanico nostro idiomate reddita: secundo Francico; tertio Italico; quarto (si dari potuit) Hispanico, aut Anglico: eaque loco subiecti (ut ita loquar) quod deinde per uaria synonima cum Arabum, tum Hebraerum, Graecorum, Lybicorum, Medorum, \& Latinorum, Chymicorum, \& Pharmacopolarum explicatur.

El propósito de los dos repertorios incluidos en Onomastica, dirigida a estudiosos de la filosofía natural y de los textos de Paracelso, era explicar los significados oscuros de las voces de estas obras y hacerlas menos herméticas: la ciencia no puede progresar si no se aleja la oscuridad de las palabras con que ésta se explica ("ne vocabulorum obscuritate deterriti, artem quoque ipsam floccipendant”, a4r). Para ello, lo importante era fortalecer las lenguas vulgares, y un vocabulario era lo más adecuado.

\section{ESTRUCTURA DE LA OBRA Y CONTENIDO LÉXICO}

Pasados los preliminares, que llevan numeración propia en romanos, comienza el primer léxico, que es el que recoge los sinónimos con que podía designarse una sustancia mineral, vegetal o animal (Onomasticon primum, philosophicum Medicum, variis linguis $\mathcal{E}$ synonimis perspicuum: antehac nunquam evulgatum). La primera lengua de la que se recopilan las diferentes denominaciones con que es designada esa sustancia es el alto alemán; a continuación, como se ha indicado anteriormente, se da su equivalencia en latín, francés y, siempre que es posible, en italiano, español e inglés. 
Este primer léxico ocupa las primeras 381 páginas y está dividido temáticamente en las siguientes partes:

1) De Gemmis \& Metallicis (p. 1).

2) Sequuntur animalia, quorum usus est in Officinis (p. 51).

3) Sequuntur aromata \& species (p. 99).

4) Sequuntur frumenta \& legumina (p. 110).

5) Sequuntur herbae radicesque Officinarum (pp. 122-381).

De todos los apartados, el último es claramente el más amplio, pero su epígrafe no recoge con exactitud su contenido, pues, junto con las voces de plantas, se incluyen de modo ocasional voces de animales (serpiente, gusano della seda, escarauaio pelotero, comadreja, grajo, tórtola, paloma, etc.), crustáceos, frutas, aceites y otras sustancias usadas entonces con fines terapéuticos (como azeyte de canincro; holleio della calebra [sic] -la despouille d'un serpent-, la piel de la serpiente; casilla-i.e. 'cajilla'- de la almeia, saliua, sangre de vaca, hiel, etcétera).

\section{PHILOS. MEDICVM. S}

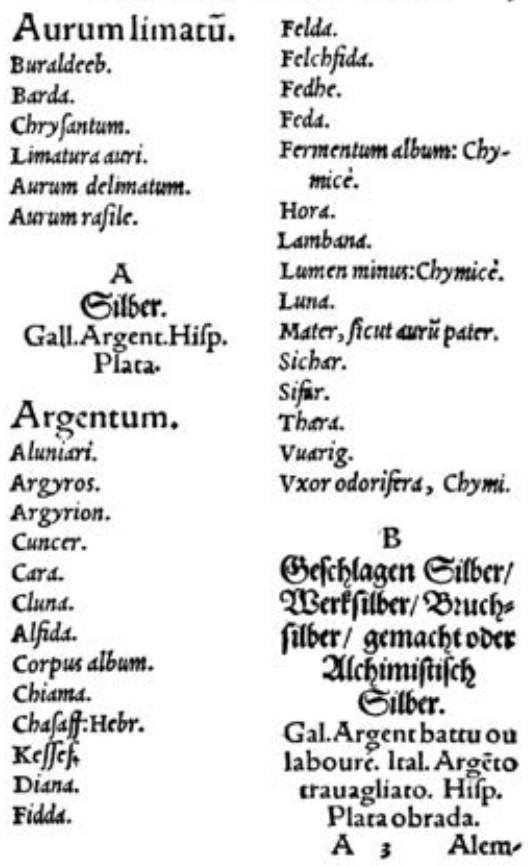

Imagen 1. Página del "Onomasticum primum” de Toxites. 
La ordenación general de las voces es temática, en forma de nomenclatura, como acabamos de ver con las cinco partes señaladas, pero el orden en el interior de cada parte es peculiar. Por un lado, se hacen pequeños subapartados de series cuyos componentes siguen la correlación de las letras del alfabeto: $\mathrm{A} / \mathrm{B} / \mathrm{C} / \mathrm{D} / \mathrm{E} \ldots$.. lo que indicaría una cierta relación entre los términos que forman: así, por ejemplo, entre las páginas 75-93 se sigue una ordenación de arbustos / árboles / frutos, y en las páginas anteriores, sustancias minerales, como:

A Stahel. Gal. Acier. Ital. Acciaio. Hisp. Acciel, vuel Azerro. Angl. Stele.

B Eisen. Gal. Fer. Ital. Ferro. Hisp. Hierro. Angl. Yron.

C Schleisstein/ Wezstein. Gal. Queue ou Pierre à aguiser. Ital. Cote. Hisp. Aguzadera. Lapis molaris.

D Zigel. Gal. Tuile ou brique. Ital. Tegola de matone. Hisp. Teia o ladrillo.

E Ofenra[u]m oder Aschenglunst... Gal. Ce que pend en la fournaise come vne grappe de raisin: ou la cendre chaude du telle grappe \& d'erain, ou le pierre d'erain. Italicè, Sasso di bronzo: ò favilla di Tutia. Hisp. Hollín de la hornaza de cobre (pp. 31-33).

No obstante, no siempre parece seguirse la estructuración. En página 99, el término A/ chastaigne d'Indie (castaña de Indias) está separado de B por el comienzo de la siguiente sección, Aromata y Species: B/ le (sic) fleur de Muscat, macis. Hispan. Macas, macias: todo parece indicar alguna inconsistencia o la falta de una revisión final (si no la intromisión del impresor), pero también hay que ser conscientes de la dificultad que supondría reunir las voces en varias lenguas de diferentes obras.

Por otro lado, aunque la voz alemana es la que abre cada apartado, en tipografía gótica, se destaca también con una tipografía mayor la primera palabra que sigue tras las equivalencias vulgares (normalmente la equivalencia latina: en la Imagen 1, Argentum). Es esta voz latina la que realmente determina el orden conductor hasta el final del vocabulario, probablemente por ser el latín la lengua de cultura internacional y porque era la lengua común en todos los repertorios utilizados. Sin embargo, aunque es la que determina el orden alfabético desde el principio de la obra hasta el final, éste no es estricto: sólo se usa la primera letra de cada palabra (como ocurría en las obras lexicográficas de comienzos del siglo XvI, aunque lo usual sería 
que se alfabetizara teniendo en cuenta las dos primeras letras de la palabra), y el volumen de voces tampoco está compensado: el $75 \%$ del primer vocabulario de Onomastica recoge palabras que comienzan por la letra A-y sólo en las páginas finales (pp. 306-382) incorpora del resto del alfabeto. Esto hace pensar que el autor vio la magnitud de su trabajo y quiso atajarlo para las voces de la $\mathrm{B}-\mathrm{V} / \mathrm{Z}$ de una manera rápida.

Veamos algunos ejemplos de estos subapartados correlativos especificando la equivalencia castellana (la palabra latina destacada tipográficamente es la que determina, de forma aproximada, el orden de las voces en la obra, pp. 233-240):

A Gros Nessel. Hisp. Hortiga. Acalise...

B Klein Nessel. Hisp. Hortiga [menor]. Acicula...

C Wullfraut. Hisp. Verbasco o gordolobo. Auidon...

D Fenchelsurt. [Sin equiv. hisp.]. Alcakar...

E Gichfraut. Hisp. Rosa montes, rosa albardera. Assurex...

F Gichtrosen. La flor de Rosamontes, y rosa albardera. AzInoRUM ROSA...

A Wilt Moren. Ital. Pastinaca selvestre. Ariostaphilon...

B Pilsternat. Hisp. Sanahoria [sic] blanca. Algazar...

C Wilder Ziedwar. Hisp. Nueze blanca, anorca. AlPHestera...

D Wild vorgel. Ital. Procacchia selvestre. ABoANIFE...

La única marca de especialidad que se usa en la obra es la de "Chy./Chym./Chimi./Chymicè" (con todas estas variaciones), pero incluso ésta aparece con poca frecuencia.

Los nombres recopilados en el primer vocabulario muestran la diversidad con que se designaban ciertos elementos en las obras que circulaban por la Europa del siglo xvi. Por ejemplo, para el estaño se dan los siguientes términos:

Zinn. Gall. Estain. Ital. Stanno. Hisp. Estano [sic]. Alcorza. Asserebum. Plumbum album. Alupnus. Alkalat. Arencho. Acaleum. Alchalai. Alleoch. Arlumba. Almila. Alenet. Baffor. Casac. Cazir.

Para la plata, los siguientes:

Silber. Gall. Argent. Hisp. Plata. Argentum. Aluniari. Argyros. Argyrion. Cuncer. Cara. Cluna. Alfida. Corpus album. Chiama. Chasff: Hebr. Kessef. Diana. Fidda. Felda Felchfida. Fedhe. Feda. Fermentum album: Chimicè. Hora. Lambana. Lumen minus: 
Chymicè. Luna. Mater, sicut aurum pater. Sichar. Sifur. Thara. Vuarig. Uxor odorifera, Chymi.

Posteriormente, se añaden los términos referidos a la plata obrada, en las diferentes lenguas, y lo mismo para la plata limada y para la hez de plata (lo que muestra cierto grado de especialidad de la obra y la acerca claramente a la lexicografía especializada o vocabularios terminológicos, al incluir tras los generales otros términos más específicos):

Schactè/Schaum oder Abfamete des Metals. Gal. L'escume \& l'ordure du Metal. Hisp. Hez de Plata. Scoria argenti. Helcysma... Argenti retrimentum. Argenti recrementum. Spurcicies metalli. Foeces argenti...

De la misma manera se especifican los equivalentes del oro, separadamente de los del oro maleable o labrado y de los de la limadura de oro, y así con otras sustancias. Por ejemplo, esa riqueza terminológica se da también en las voces de plantas, como perejil (con otra entrada perexil nigro, o salvaje, posteriormente):

Hausepfich/ Mark / Gleis / Peterlin / Petersilie

Gall. Persil. Ital. Petrosello, apio domestico. Hisp. Perexil. Apium. Alcharfas. Alcarapse. Carufu. Cyfa. Curaff. Gaxit. Harps. Kaspar. Karff. Karasum. Narn. Oreosilinum. Petroselinum domesticum. Querrasum. Sazonech. Selinon/ Seiuon. Sipui. Hipposelinum. Ipposelinon. Xilinon.

Gros Peterlin/ Wild Petersilien/ Bergpeterlin. Gall. Persil sauvage.

Ital. Petrosello grande. Hisp. Perexil nigro [sic]. Alexandrum. Anoni. Agrioselinum.

Alistacabum nigrum. Busellinum. Fista solida. Grielon. Heliosellinum. Eleoselinon. Ipposellinum. Macedonicum selinum. Mudnan. Mufran. Oreosellinum. Obfastruin. Oxixatrum.

Petros agreste. Rurafra. Smonum. Smirnion. Suumen.

Desde el punto de vista cuantitativo, los términos recogidos en este primer vocabulario son en total, si nuestro cómputo no es errado, 775 , de cada uno de los cuales se pueden dar en torno a 8-20 sinónimos en otras lenguas: siempre se da el término en alemán y en latín, que cuenta habitualmente con muchas otras denominaciones; la lengua francesa está práctica- 
mente siempre presente, salvo en reducidos casos; en cuanto a la lengua española, en este primer vocabulario de Onomastica I se dan equivalencias para 465 entradas, lo que supone un $60 \%$ del total de éstas. Ahora bien, como en muchas de esas entradas se da más de un equivalente español, podemos establecer un total de, aproximadamente, 500 términos en lengua espanola. Se trata de un número considerable para su época y para voces de especialidad, referidas a plantas, animales y minerales en su mayor parte, lo que realza la importancia de la obra y su empleo en los estudios de léxico.

A continuación se ofrece una muestra de algunas voces castellanas que incluye este primer vocabulario. Realizamos primeramente una tabla con los términos españoles extraídos de las primeras treinta páginas de Onomastica I (respetamos la ortografía de la época, pero se moderniza la acentuación) y luego dos párrafos de otros apartados (ponemos en ocasiones entre paréntesis la equivalencia francesa o latina cuando puede ayudar a la comprensión de los términos) ${ }^{16}$ :

\section{TABLA 1}

Muestra de voces españolas, con sus equivalentes en otras lenguas, de Onomastica I

\begin{tabular}{llll}
\hline \multicolumn{1}{c}{ Término español } & \multicolumn{1}{c}{ Francés } & \multicolumn{1}{c}{ Alto alemán } & \multicolumn{1}{c}{ Latín/otras lenguas } \\
\hline perla & marguerite & Perlein & albula, perla alba \\
asmaralda $($ sic $)$ & esmeraulde & Schmaragd & $\begin{array}{l}\text { alferuzegi, lapis } \\
\text { smaragdus }\end{array}$ \\
& & Segelstein od' & calamita, lapis \\
piedra ymán & aimant & Zeilstein & magnetis \\
& & Gold & aurum, lapis aureus \\
oro & or & Silber & argentum \\
plata & argent & &
\end{tabular}

${ }^{16}$ En este primer vocabulario (Onomastica I) se dan otras entradas relativas al oro, aunque estas otras no incluyen su equivalente en español: aurum coctum (Gall. L'or qui est mis en oevre); aurum limatum (Gall. Or limê). Sí aparecen, sin embargo, en el vocabulario segundo (Onomastica II, p. 404), bajo la voz aurum: "[aurum] purum, Gall. L'or qui n'est pas en oeure. Ital. Oro puro $\mathcal{E}$ schietto. Hisp. Oro que aún no está labrado. Item aurum obryzum, \& excoctum, recoctum... Gall. Or pur. Ita. Oro fino. Hisp. Oro recozido, sin mezcla. Item aurum netum, in fila ductum... Gall. Or filé. Ital. Oro filato. Hisp. Oro hilado". Es patente, pues, la dependencia entre los vocabularios, como ya vimos en nota 10 al hablar de las remisiones de uno a otro (voz litargirio). 
TABLA 1

Muestra de voces españolas (continúa)

\begin{tabular}{|c|c|c|c|}
\hline Término español & Francés & Alto alemán & Latín/otras lenguas \\
\hline plata obrada & $\begin{array}{l}\text { argent battu ou } \\
\text { labouré }\end{array}$ & $\begin{array}{l}\text { geschlagen Silber } \\
\text { / Werksilber }\end{array}$ & $\begin{array}{l}\text { alembus, argentum } \\
\text { malleatum }\end{array}$ \\
\hline hez de plata & $\begin{array}{l}\text { l'escume \& } \\
\text { l'ordure du metal }\end{array}$ & $\begin{array}{l}\text { Schlackem/ } \\
\text { Schaum oder } \\
\text { Abfamete des } \\
\text { Metals }\end{array}$ & $\begin{array}{l}\text { scoria argenti, } \\
\text { spurcicies metalli }\end{array}$ \\
\hline cobre & cuyvre brusle & $\begin{array}{l}\text { gebrant Kupfer / } \\
\text { gebranter Messing } \\
\text { / gebrant Erz oder } \\
\text { Kupfererz }\end{array}$ & $\begin{array}{l}\text { azimar ustum, } \\
\text { cuprum ustum }\end{array}$ \\
\hline esquama del cobre & l'escume d'erain & $\begin{array}{l}\text { Abgang } \\
\text { od'Schabet vom } \\
\text { Kupfer oder Erz/ } \\
\text { Kupferschlag }\end{array}$ & aconis patromos \\
\hline cardenillo & la rouillure d'erain & Erzrost / Erzblust & $\begin{array}{l}\text { alchabrusi, ferrum } \\
\text { rubeum }\end{array}$ \\
\hline estano (i.e. estaño) & estain & Zinn & $\begin{array}{l}\text { alcorza, plumbum } \\
\text { album, stannum }\end{array}$ \\
\hline plomo & plomb & Blei & alabrem \\
\hline azogue & argent vif & Quecksilwer & argentum vivum \\
\hline aluayalde & fard & $\begin{array}{l}\text { Bleiweis oder } \\
\text { Lotweis }\end{array}$ & albaiet, flos plumbi \\
\hline azufre & soulfre & Schwebel & alkibric, ignis terrae \\
\hline bermellón & vermillon & $\begin{array}{l}\text { Zinober / } \\
\text { Draehenblut oder } \\
\text { Bergzinober }\end{array}$ & $\begin{array}{l}\text { abitabiel, } \\
\text { cynabrium }\end{array}$ \\
\hline oropimiente & orpin & Arsenik & $\begin{array}{l}\text { auripigmentu, } \\
\text { arsenicum citrinu }\end{array}$ \\
\hline alcohol & antimoine & $\begin{array}{l}\text { Spisglas / Bleiklar } \\
\text { oder Todblei }\end{array}$ & $\begin{array}{l}\text { antimonium, } \\
\text { plumbum } \\
\text { minerale, queheli } \\
\text { hispanici, terra } \\
\text { hispanica }\end{array}$ \\
\hline almartaga & $\begin{array}{l}\text { escume de cuyvre, } \\
\text { or ou argent }\end{array}$ & $\begin{array}{l}\text { Goldglid oder } \\
\text { Bled oder } \\
\text { Kupferscheid }\end{array}$ & $\begin{array}{l}\text { asmartalla, } \\
\text { litargirium, spuma } \\
\text { aur }\end{array}$ \\
\hline caparrosa & couperose & $\begin{array}{l}\text { Schwarz } \\
\text { Kupferwasser oder } \\
\text { Kupferrauch }\end{array}$ & $\begin{array}{l}\text { atramentum } \\
\text { griseum, terra nigra }\end{array}$ \\
\hline
\end{tabular}


TABLA 1

Muestra de voces españolas (concluye)

\begin{tabular}{llll}
\hline \multicolumn{1}{c}{ Término español } & \multicolumn{1}{c}{ Francés } & \multicolumn{1}{c}{ Alto alemán } & \multicolumn{1}{c}{ Latín/otras lenguas } \\
\hline alumbre & alun & alaun oder alat & alumen \\
sal azedo & sel sur, ou aigre & Sorsalz & atinca, sal alkali \\
alatrón & sel depierre ou du & Salpeter, Bauksalz, & auatroy, sal nitri, sal \\
& mur & Stunmmsalz, & vugaru (sic) \\
& & Wandlblüst & \\
cal por regar & chaulx vive & Ledder Kalch, & almura \\
& & Rauher oder & \\
& & ungeldscheter & \\
& & Kalch, & \\
& & vungeblüheter & \\
cal & & Kalch & \\
tierra sellada & Kalch & avora \\
& terre seellé de & Gesigelt Erd & albohera lutum, \\
& Lemnos en & / Mörlaimen & terra Sarracenica \\
& Turquie & / Türkischer & \\
& & Lemnnischer & \\
& & laimen & \\
& & & \\
& & &
\end{tabular}

Siguen otras voces como huego (i.e. fuego); horno o hornaza; almagra (fr. croye ou terre rouge); piedra esponia; agua; agua de la mar, agua salada; agua de ceuada; arzilla; hiendo de cavallo; es$t[i]$ ércol del buey; lexía de ceniza; limo; betún iudiego; azeite d'almendras; hollín dela hornaza de cobre, perla; asmaralda ( sic); coral; piedra ymán; oro; oro maleable o labrado; limadura de oro; plata; plata obrada; plata limada, alforza; hez de plata; latón; cobre; estano (i.e. estano); plomo; azogue.

En el apartado de "herbae radicesque Officinarum" se incluyen acoro; grana roxo (fr. grains rouges ou d'escarlat; lat. coccus baphicus, grana tinctorum); raýze de rosa montes (fr. radice de penoesne, lat. radix peonie); runia (fr. garance); semiente de gatillo casto; pimiento; sauz gatillo (lat. piper eunuchorum); alcapparas (sic); azufecifa; romero; abrotono, herba lombriguera (lat. abrothanum agreste); amoradux, axedrea, maiorana; magaza (fr. athanasie); yerva cidrera, yerva avegera; cárcamo alacor (i.e. cártamo, alaçor), semiente de papagayos (fr. semence du saffran bastard $\mathcal{F}^{2}$ sauvage); hiquera del infierno (fr. espurge grand); goma d'alerze (fr. gomme clair du cedre); goma d'yedra (fr. gomme ou distillant de lierre); xara; encienso; linaloe; ciprés; enebro (alabel, arbor iuniperi); lauriero, laurel; palmera (lat. arbor dacti- 
li); cerezo silvestre (lat. arbor cornea); robre (i.e. roble); álamo; moral; genestra, hiniesta, retama, sparto (lat. arbor montana); granillo del enebro, etcétera.

Como se puede ver, estos términos se refieren tanto a sustancias minerales usadas en compuestos (como mercurio, azogue, azufre, bermellón, alcohol, alumbre, plomo, etc.), como a sustancias animales y vegetales (cola, engludo [sic], est[i]ércol del buey, lexía de ceniza), a animales y plantas (águila, camello, osso, halcón o azón (sic; i.e. azor), abubilla o popa, cuguiada (i.e. cogujada), cotovía; enebro, linaloe, granadero, robre, retama sparto, genestra/ hiniesta...), a especias (nuez d'especia, regaliza, hongo de abeto, sangre d'erizo) y a hierbas y raíces medicinales (romero, axedrea, magaza, higuera del infierno, etcétera).

Aunque lógicamente encontramos términos generales, otros son especializados, y es aquí donde radica la relevancia de esta obra, pues son escasos los vocabularios especializados en el siglo XVI (salvo en el ámbito náutico y médico, entre los que se insertaría esta obra). Algunos ejemplos serían: aluayalde (carbonato de plomo o blanco de plomo, usado en pintura) y sus denominaciones en otras lenguas: oximilium/ oximalium, minium album, flos plum$b i$; alumbre (alumen, azeb, alumen de rocca, alumen de Bononia, lapis hardis, sulphur album), lo que hoy se denominaría sulfato doble de alúmina y potasio, empleado en medicina y en la industria; el agua mercurial, oro hilado, esquillo, cal porregar (= 'cal viva'), berueco (i.e. berrueco 'lesión con aspecto de verruga que aparece en el iris de los ojos', según el DLE), etcétera.

Pensando en el uso terapéutico de la época, más que en la escatología, se designan los diferentes tipos posibles de orina (urina del hombre, urina de mozo, urina de perro, urina de vaca, urina de buey) o de estiércol usados entonces (hienda de gorrión, mousse de chesne, sterco del celega); capaión del perro (sterco del cagnuola, fiante ou merde du chien). También se dan diferentes tipos de aceites, como el azeyte de laurel, azeyte de azucena, azeyte de violeta, azeite de rosa, azeyte de canincro o azeyte de vua [= 'uva'] aún no matura (sic).

El segundo vocabulario es más breve: abarca sólo cien páginas (pp. 383-490) y contiene 636 voces, de las cuales sólo 87 contienen alguna equivalencia española. Su técnica lexicográfica es menos depurada: como tiene el propósito de explicar voces o pasajes oscuros, muchas equivalencias no son tales, sino traducción de frases, como veremos en los ejemplos. No obstante, también incluye términos. Está alfabetizado de manera estric- 
ta por la voz latina; tras el lema sigue su definición o, más bien, explicación en latín y su traducción al alemán. Se da con frecuencia su traducción al francés y sólo ocasionalmente al italiano o español.

Como es un diccionario basado en las voces de las obras de Paracelso, se recogen muchos relacionados con la alquimia, la astrología y la filosofía natural, como por ejemplo los siguientes:

Arcanum, res est secreta, incorporalis atque immortalis, quae ab homine cognosci non potest, nisi per experientiam: est enim virtus cuiusque rei, quae millesies plus operatur, quam res ipsa. Gall. C'est le secret incorporel, immortel, que l'homme sans experience ne peut point cognoiste: car c'est la vertu de touts choses qui faict plus que la chose mesme.

Bruta, est vis quaedam influentiae coelestis, quae per animalia bruta manifesta fit... Gall. Quelque efficace cachee de l'influence celeste, qui se montre par les bestes.

Influentia est, quando imaginatione \& mente superiorum syderum, atque planetarum naturam ac virtutes in nos attrahimus... Gall. C'est quand nous par imagination forte, nous attirons la vertu des planetes.

Chaomantia, quando per aerem aliquid futurum praeuideri ac praedici potest... Gall. Quand on pronosticque quelque chose de l'accidents Eै l'aduenemens au l'air.

Cherio, est elementorum externorum accidens, siue natura caliditatis, aut frigiditatis, per quam omnes aegritudines sanantur... Gall. L'accident des elements exterieurs, ou la nature du chauld $\mathcal{E}$ froid.

Chiromantia, est ars, quae non tantum hominis naturam ex manuum lineis cognoscit, sed etiam planetarum, \&c... Gall. C'est art non seulement endouiuant hors la main, mais aussy les planetes.

Destacan, no obstante, los términos referidos a metales o sustancias utilizadas con fines químicos. Damos algunos ejemplos:

Acetum Philosophorum, id est, Lac uirginis, siue aqua mercurialis, qua metalla solutuûr. Hydor sophorum, bedy hermu. Philosophischer essig Jungfraumulch, ein Mercurialwasser/ das die Metallen aufloeset. Gall. Eau ou aigue Mercurialle, souldant les metaulx. Ital. Acqua del mercurio. Hisp. Agua mercurial. 
Aes: est proprie Cuprum, sed interdum à nonnullis usurpatur pro auro, argentoque non fuso, sed à natura excocto, ex quo olim monetam cuderunt. Eigentlich heist es Kupfer / etlich brauchen es für lauter gold oder silber ertz / welchs noch nicht gegossen / sonder von Matur ausbereit ist / daraus man erwan münz geschlagen: Gall. C'est proprement cuyure, mais quelcuns interpretent Or $\mathcal{E}$ argent, qui n'est pas encore mis em oeu[u]re. Ital. Oro $\mathcal{E}$ argento schietto. Hisp. Oro Ẽ plata que aún no está labrado.

Alchahest, est mercurius praeparatus in medicinam epatis. Gall. Argent vif preparatif pour le foy. Hisp. Argento bivo d'el hígado. De hoc vide librum de viribus membrorum.

Carena, vicesima quarta parts guttae, der 24. theil aines tropffè: Gall. La vint quatrieme partie d'vne goutte.

Grauus marmor, vel porphirius, porphyrites, marmor porphyreticus, rubius... Gall. Marbre rougeatre. Ital. Porfido. Hisp. Mármol de porfido.

Koboltum vel Cobaltum nigrum, interdum etiam fere cineritium, funditur \& laminatur, sed colore caret metallico, propterea fixum non est, sed meliora metalla secum per fumum abducit. Est Cadmia, lapis é quo aes fit, medicinae vtilis, lapis aerosus, Climia, Cathimia, lapis calaminaris... Gall. Pierre d'ou on faict erain. Hisp. Hollín de la hornaza de cobre.

Luna compacta, est argentum fixum, siue aurum album. Fix Silber/ oder weisz Gold/ Gall. Or blanc.

Otros muchos términos incluidos se refieren al ámbito médico:

Botium, apostema, siue escrescentia in gula, veluti struma. Ein geschwaer oder bergewaechs inn der gurgel als ein fropf. Gall. Inflation ou apostume dedans la gueule, comme escrouelle. Ital. Gonfiadura de gola al modo de gozo. Hisp. Hinchadura de tragadero à puerca y lamparón $n^{17}$.

Bothor vel Cossi, apostemata sunt in facie alba \& parua, papulae faciei, boae, eczesmata, pythedones. Seirlin oder weis blaeterlin

17 Para mayor detalle de las voces puerca y lamparón, véase infra el apartado "Análisis de algunas voces castellanas". 
un angesicht/ Gall. Petites verolles blancs au face, vessies. Ital. Variole vesica, ampolle di faccia. Hisp. Lobanillo, pestilla de rostro y cara.

Butiga siue guta ruonia, vel rubea, est inflatio totius faciei, Das gewülf. Gall. Inflation de toute la face.

Cenigdam vel cenigotam, est instrumentum quo craneum in epilepsia perforatur, ein Instrument da mit man die hirnschaln $\mathrm{o}^{\mathrm{e}} \mathrm{f}$ net. Gall. Instrument pour ouurir le tez de la teste.

Colica, tartarus est resolutus in instestinis morbusque fixus, colicus dolor coli intestini morbus, colica diathesis... Gall. La colique passion. Hisp. Cólica.

Consolidatura, medicamentum cicatricem ducens, epuloticon, incarnans, sarcoticon... Gall. Remede pour fermer la playe, medicine incarnatiue. Ital. Remedio per choider Ẽ serra la piaga, in carnatiuo. Hisp. Emplastro para cierrar o encarnar llagas, E $\mathcal{F}^{2}$ ayuntar los huessos.

Coostrum, media pars septi transuersi, seu diaphragmatis, Das mittel des Leibsleist. Gall. Vne peau separant les parties interieures. Ital. Pelle che separa le parti interiori. Hisp. Pelleia apartadera de las entrañas, y otras partes.

Lumbrici, etiam in homini reperiuntur in omnibus prope membris: quorum veriae species sunt: longi, breues, lati, graciles \&c. $\mathrm{Spu}^{\mathrm{e}}$ lwürm/Bauchwürm. Gall. Ver au ventre d'homme. Hispanic. Lombriz. Sunt etiam lumbrici teretes, lati \& longi, falciae dictae \& taeniae, helminthes plateaiae, ceiriae.

Macula oculi, suffusio, hyposchysis, hyposchyma, cataracta, Ster$\mathrm{nfel} / \mathrm{schemerina/Gall.} \mathrm{Cataracte} \mathrm{de} \mathrm{l'oeil.} \mathrm{It.} \mathrm{Suffusione} \mathrm{del} \mathrm{occhio.}$ His. Cataracta de oios.

Phtisis. Gall. Ethique. Ital. Tisica. Hisp. Trefe. Alio qui atrophia ferè idem,nutrimenti cessatio, ex timore aut nimia auiditate existens, das abnes men/abfallen vom leib... Gall. Quand on est en chartre. Hisp. Entonces que el hombre está malo de tísica.

Otros, al ámbito natural:

Caliette, fungi flaui in plantis iuniperorum. Gelbe schwemlein an den wechholder stauden. Gall. Les champignons des geneures. Ital. Fungo del ginepro. Hisp. Cogumelos d'enebro. 
Cheiri, multifariam etiam sumitur. Nam, \& de herba dicitur, \& cum de metallis loquimur argentum viuum dicitur, quidam vero aliter accipiunt: alii aurum potabile esse volunt, alii Antimonium... Gall. Les herbiers appellent ainsi quelque herbe, mais aupres les metalliers cest argent vif, ou or potable, ou antimoine.

Fabiola, flos fabarum, bonen plüst. Gall. Le fleur des febues. Ital. Il fiore di baccieli. Hisp. La flor d'haua.

\section{ANÁlisis de ALGUNAS VOCES CASTELlanas}

Lo transcrito hasta aquí demuestra que la obra ofrece un claro interés lexicológico para la lengua española, como un testimonio más, hasta ahora no considerado, de muchas voces generales y de especialidad.

Un análisis exhaustivo que tuviera en cuenta todo el léxico de los dos vocabularios y lo comparara con el ofrecido en otras obras cronológicamente cercanas, lexicográficas o no, podría ofrecer dataciones complementarias a las hasta ahora disponibles y establecería el grado de innovación o dependencia de éstas, pero para ello sería necesario elaborar un corpus de las voces de todas ellas, lo que no es viable para este artículo, pero sí, por ejemplo, para una memoria de maestría. Animamos pues a realizarlo, con textos renacentistas y barrocos, tal y como se hizo con textos manuscritos del siglo xv en el Diccionario espanol de textos médicos antiguos (abreviadamente DETEMA) o como elaboramos nosotros parcialmente en la monografía Hacia una flora universal (2010).

En tanto, en el presente estudio hemos realizado un primer análisis de este léxico que mostrará su relevancia. Para ello hemos recurrido al diccionario etimológico de Corominas, al DETEMA, CORDE, Nuevo tesoro lexicográfico (s. XIV-1726) de Nieto y Alvar (NTLE), Nuevo tesoro lexicográfico de la lengua española de la Real Academia (NTLLE) y Corpus del Nuevo diccionario histórico del español $(\mathrm{CDH})$.

De este análisis, del que veremos ejemplos a continuación, se deduce que, si bien buena parte de las voces se incorpora ya en otras obras de la época o aun en anteriores, la de Toxites debería incluirse entre las primeras fuentes, pues en ocasiones los corpus recogen documentaciones precedentes, pero, en muchos casos, de manuscritos. Su obra, además, sería una fuen- 
te lexicográfica destacable entre los diccionarios publicados en el siglo XVI que dan cabida al español.

Así, Onomastica está entre los impresos más tempranos que atestiguan determinadas voces: almártaga, con el sentido de 'litargirio, óxido de plomo' (o 'escoria de plomo mucho molido', según el DETEMA, p. 80), sólo se documenta en otra obra del siglo xvi (De re metallica de Bernardo Pérez de Vargas, 1569) y, posteriormente, en los Discursos medicinales (16061611) de Juan Méndez Nieto, en el Arte de los metales (1640) de Álvaro Alonso Barba, así como en Fontecha (1606). Lexicográficamente, incorporan la voz el vocabulario español-latino de Nebrija (ed. 1520), el comentario al Dioscórides por Laguna (1555), el vocabulario de Palmireno (1569) y el diccionario de Cristóbal de Las Casas (1570: "almártaga: litargiro"); otros, posteriores, como los de López Tamariz (1585), Percival (1591), Palet (1604), Oudin (1607) y Covarrubias (1611) también la incluyen, pero, además de por su temprana documentación, la de Toxites tiene el mérito de ser una obra impresa en el ámbito centroeuropeo, fuera de las áreas de influencia española directa.

En cuanto a la voz litargirio, que se recoge en Onomastica II (cf. nota 10), el CORDE la documenta en al menos siete obras anteriores a 1574, todas ellas médicas, como la traducción del Tratado de cirugía de Guido de Cauliaco (1493), el Sumario de la medicina con un compendio sobre las pestíferas bubas (1498) de Francisco López de Villalobos o el Tratado llamado Fruto de todos los autos contra el mal serpentino (1542) de Ruy Díaz de Isla, pero prácticamente son todas manuscritas, salvo De re metallica (1569) o los Diez privilegios para mujeres preñadas (1606) de Juan Alonso y de los Ruyzes de Fontecha y otras posteriores, ya impresas (y no se documenta de forma abundante: tras 1606, el CORDE recoge la siguiente documentación en 1791, en los Anales del Real Laboratorio de Quimica de Segovia). Lexicográficamente, según el NTLE y el NTLLE, sólo se recoge anteriormente a Toxites en Nebrija (1545) y en el comentario de Laguna al Dioscórides (1555); siguen luego pocas documentaciones: Minsheu (1599), Fontecha (1606) y el diccionario hispano-francés de César Oudin (1607: "l'escume ou ordure de l'argent dont on se ser és medecines"); Covarrubias (1611) lo define como "vngüento de lytargyros, que vale spuma argenti, especie de betún mineral"); en los diccionarios académicos se incluye desde 1780 , pero remiten a almártaga. 
La voz oropimiente (arsenicum citrinum), aunque está documentada en el manuscrito Compendio de cirugía (1481) de Guido Lanfranc de Milán y en la Suma de la flor de cirugía (s. Xv) (cf. DETEMA, p. 1153), se localiza en el CORDE en tan sólo dos obras anteriores a Toxites (la traducción de El libro de proprietatibus rerum, de Bartolomé Ánglico, por fray Vicente de Burgos, de 1494, y otro manuscrito de 1495, la traducción española del Lilium medicinae de Bernardo de Gordon) y en otras dos posteriores: en el vocabulario médico de Juan Alonso y de los Ruyzes de Fontecha, en los Diez privilegios para mujeres preñadas (1606), y la Historia del Nuevo Mundo (1653) de Bernabé Cobo. Lexicográficamente, según el NTLE, con grafía oropimiente u otras (oropimente, oropimiento) se documenta en Nebrija (1495?, oropimente o jalde), en el Vocabulista arábigo de Pedro de Alcalá (1505), en la obra de Amato Lusitano (1553), en el comentario al Dioscórides por Laguna (1555), en Palmireno (1560), Fragoso (1566), Junius (1569), Palmireno (1569), Las Casas (1570) y en otros diccionarios posteriores, como el de Hornkens (1599, con la variante almáitiga), el Tesoro de Covarrubias (1611), el multilingüe de Minsheu (1617) y el bilingüe de Franciosini (1620).

La voz tarma, "especie de carcoma que roe la madera", según Terreros (1788), se incluye en Toxites (1574) como un tipo de xilófago (ya citamos la voz supra-cf. "Onomastica. Descripción de la obra"-al hablar de la escasa presencia de equivalencias inglesas: "Assaliae, vermes sunt intra asseres crescentes... xylophagi. Gall. Ver de bois. Ital. Tardo, verme di legno. Hisp. Tarma. Angl. à Tymber vuorme"). No se trata de la primera documentación: el NTLE la registra a partir del vocabulario políglota de Garonus (1526: lat. tarmus, ital. le tarme, fr. ver de boys, esp. tarma); de aquí pasó al vocabulario Quinque linguarum de 1534, con las mismas equivalencias, y luego la menciona Junius (cossus... teredo..., termex..., tarma). Minsheu (1599) la define: "tarina [sic] ... a worme that eateth timber", al igual que César Oudin (1607, tarma: "ver qui ronge le bois"). Vittori (1609) lo copia, Franciosini (1620) y Trognesio (1639) también. Luego, Antoine Oudin (1647) especifica: "tarma, polilla", y Mez (1670), "tarma, ein Holtzwurm"; Stevens (1706) copia a Minsheu: "tarina, a worm that eats timber". La de Toxites (1574) resulta, pues, un testimonio temprano no considerado.

Las voces puerca y lamparón, ya mencionadas anteriormente, son términos específicos de medicina; aparecen en tratados de cirugía, como el Compendio cirúrgico, útil, y provechoso a sus pro- 
fessores (1719), de Diego Antonio de Robledo, en que se describen así (modernizo la ortografía):

Las escrófulas, estrumas o lamparones son nombres que en diversas lenguas significan una misma cosa, aunque algunos dan diferencia entre escrófulas y estrumas en ser los unos lamparones con más dureza que los otros; pero así las escrófulas como las estrumas se llaman en nuestro idioma castellano lamparones o puercas, con que debajo de cualquiera destos nombres se puede tratar destos tumores, y así con el nombre lamparón trato dellos. Lamparones, según Daza, son "unos tumores duros, que nacen en las partes glandulosas, y de ordinario en el pescuezo, hechos de humores crasos secos, y endurecidos, de tal suerte que parecen carne blanca, los quales están encerrados en una túnica hecha de la misma materia, y son inobedientes a la resolución, y suelen crecer fácilmente".

Lexicográficamente, la voz puerca-con este sentido médicofigura desde Nebrija (1495?: "puerca pequeña, sucula, ae; puercas como lamparones, scrofulae, arum"; Nebrija 1518: "choeras, adis, morbus qui latine strume siue scropha, hispane puercas"; "scrofa, ae, siue cheras, adis, latine struma, hispane puerca o lamparón”). El NTLE de Nieto y Alvar lo documenta posteriormente en la nomenclatura de Junius (1567: “choeras..., puerca o lamparón”), que será, como veremos luego, una de las fuentes principales de Onomastica. Tras esto, debería figurar la documentación de Toxites 1574, pero no es así; el NTLE no recoge otra fuente hasta el diccionario hispano-inglés de Minsheu (1599: "puercas or lamparones, kernels, swelling in the armepits or necke") y el diccionario etimológico de Rosal, 1601, más Seguin, manuscrito hispanofrancés de 1636. No hay más documentaciones en diccionarios anteriores a 1726, parece ser. Fontecha, autor de un vocabulario médico en 1606, no incluye esta voz, pero sí lamparón.

Otras voces no parecen ser habituales: por ejemplo, yerva cidrera, que sólo encuentro en una obra posterior de 1615, la Histoire generale des plantes de Dalechamps y, antes, en un manuscrito del DETEMA, el Tratado de patología general del s. Xv (aunque con la variante yerba cidrial 'toronjil, melisa'). El CORDE sólo recoge un ejemplo de época de cidrera, en una poesía de Pedro de Espinosa (1590-1650): "No falta aquí contra el azul celidro / la bazahar, dos veces estranjera, / ni la aserrada pempinela y cidro, / betónica montés, vulgar cidrera”.

Otra equivalencia de esta misma hierba, yerva avegera (i.e. abejera), sin duda así llamada por su relación con las abejas, no 
la localizo en ninguna fuente, salvo en una mención de Antonio José Cavanilles, Observaciones sobre la historia natural, geografia, agricultura, población y frutos del reyno de Valencia (1797), ya del siglo XVIII:

Esta parte de la montaña parece cortada á pico... Allí vi con abundancia la hepática, la doradilla, el culantrillo, el polipodio común y otras plantas que aman sitios húmedos y sombríos. También crece... el tenellum y crassifolium de mis obras botánicas, la abejera, y el vencetósigo.

En cuanto a la voz alcanna, que en Toxites se relaciona con guaiaco (registrado a partir de mediados del siglo XVI), el CORDE sólo ofrece tres documentaciones, dos de ellas correspondientes a los fueros de Baeza y Alcaraz (ca. 1300 y a. 1296, respectivamente, donde aparece en listados: "De alumbre, .I. meaia; De alcanna, .I. meaia"); la tercera, a Juan Alonso y de los Ruyzes de Fontecha, Diez privilegios para mujeres preñadas (1606): "Alcheten, vna simiente o hojas, semejante al alcanna". La de Toxites sería, pues, una cuarta documentación intermedia (1574) no registrada aún.

Un análisis de ciertas combinaciones también muestra la relevancia de la obra para la historia de nuestro léxico. Toxites incluye oro hilado (gall. Or filé. Ital. Oro filato. Lat. Aurum netum, in fila ductum), que se documenta en el CORDE en textos de los siglos XV-XVII, con el sentido de 'hilos de oro' ("cuerdas de oro hilado", "trenza de oro hilado", "vna onça de oro hilado, para vna borla de vn sombrero", etc.). No la encuentro en diccionarios contemporáneos, donde sí aparecen otras combinaciones; así, el diccionario hispano-francés de Palet (Diccionario muy copioso de la lengua española y francesa, 1604) señala: oro gañin (esto es, "or meslé avec argent"); oro de lata ("oripeau"); oro de tíbar ("or fin"). El diccionario de Oudin, Thresor de deux langues (1607), complementa Palet: "oro guañín de 20 quilates, or bas à 20 quarats; c'est de l'or qui a la cinquiesme partie d'argent; oro reduzido en riel, or en masse, en linots". Covarrubias habla del oro potable, "cierta inuención de alquimistas que persuaden poderse desatar este metal de manera que pueda pasar por las vías y venas, como haze el agua; no creo nada desto". Sólo cincuenta años después aparece el término en la nomenclatura trilingüe de Noviliers (1629): "oro hilado, oro di canutíglio, or de canetille, oro de cañutillo; v. cañote". Salas (1645) dice del oro 
de cañutillo: "aurum netum, filo deductum ad fisum". No parece haber más menciones de la expresión hasta Stevens (1706): "oro de cañutillo or hilado, gold purl". Parece, pues, que frente a oro de cañutillo, común en los diccionarios españoles, la inclusión de oro hilado es escasa, y Toxites (1574) daría la primera documentación lexicográfica.

Otra combinación, agua mercurial, tampoco aparece en el CORDE ni en el NTLE (sí se documenta agua fuerte, agua mala, agua manil, agua manos, agua miel, agua pie...); vino d'yerua (lat. Cardonium vinum, ex herbis compositum, Gall. Vin faict d'herbes), recogido en Toxites, tampoco se documenta entre las muchas posibles combinaciones: vino verde, vino aguado, vino rezio, dulce... Incluso Junius (1567), que recoge más de quince tipos, como vino bastardo, vino cozido, vino de su tierra, vino de manzanas, vino rebotado, vino viejo, vino de dos años, vino ordinario y de mesa, vino puro y sin agua, vino mohoso, vino de obreros..., no incluye éste de hierbas (hoy diríamos, presumiblemente, "licor de hierbas"). Sin embargo, en los comentarios al Dioscórides realizados por Laguna (1555), bajo la mención del vino, encontramos diferentes tipos, incluso los hechos con dátiles, granadas, piños, cedrino, de hysopo, "los preparados con plantas diuersas, los aromáticos y los olorosos, y los hechos de muchas suertes de yeruas".

Algunas voces recogidas por Toxites son variantes poco frecuentes, como vespo y tavarro (por 'abispa' y 'tábano', o 'abispón', según Oudin 1607) y manganilla (por 'manzanilla': documentada en Toxites, Minsheu 1599 - manginilla-, Aldrete 1606, Vittori 1609, y en algunos otros posteriores, como Franciosini y Trognesio, más en esta cita de Antonio Vázquez de Espinosa de su Compendio y descripción de las Indias Occidentales, 1629, sobre las virtudes curativas de las hierbas: "De las de España ay Romero, hinojo, orégano, ruda, culantrillo de pozo doradilla yerbabuena, yerba de Santa María, apio, peregil, torongil, culantro, poleo, manganilla, hortigas, mastuerso, berbena..., que las más de ellas son conocidas sus virtudes"); cf. CORDE.

Otras voces de Toxites no están documentadas en el CORDE ni en el $\mathrm{CDH}$, pero son probablemente erratas debidas a los impresores: recordemos que Onomastica se imprimió en Estrasburgo, de habla alemana entonces, por lo que los errores también pueden ser muestras del desconocimiento de la lengua fuera de nuestras fronteras. Así, encontramos pan cenceno en lugar de centeno, sanahoria por zanahoria-extrañamente recogida en Minsheu- o bien errores o cruces morfológicos con otras 
lenguas, como hiorno por horno, ciera por cera (con diptongación anómala), betún iudiego por judío, vinagro por vinagre, gartixa y lagardiza por lagartija-de nuevo atestiguado en Minsheu 1617, aunque este autor no es fiable; además, la falta de documentación hace improbable su uso real-, limonzas por limones, leche agro por leche agria.

A este análisis de voces concretas podría añadirse que Toxites muestra ya un cierto grado de adaptación y difusión de cultismos que habían surgido a finales del siglo Xv, como alopecia, asthma (traducido como 'dolencia de los pulmones o acezo') o botium (actual 'bocio') ${ }^{18}$. En sus obras aparecen aún como latinismos (que merecerían estudio aparte), pero son, en todo caso, testimonio del influjo de la cultura grecolatina en el enriquecimiento de las lenguas vulgares.

\section{EsTUdio DE LAS FUENTES DE ONOMASTICA}

Si lo mencionado hasta ahora se refería a la aportación de la obra de Toxites para la datación de ciertas voces, lo siguiente se refiere a sus fuentes, aunque se profundizará con ello el análisis del léxico.

Gracias a los índices parciales de una docena de obras sobre historia natural que recopilamos en el libro Hacia una flora universal (cf. Pablo Núñez 2012, pp. 233-262), hemos visto similitudes de las equivalencias españolas dadas en los vocabularios de Toxites con las voces españolas que aparecen en los varios volúmenes de la Historiae animalium de Conrad Gesner (1551 y 1566) y con las de los comentarios al Dioscórides hechos por Andrés Laguna. La edición de Amberes (1555) recoge equivalencias en varias lenguas para cada término y pudo Toxites, así, haberla usado para incorporar a sus vocabularios las equivalencias castellanas; aquí ofrecemos varias voces que mostrarían esta posibilidad, pero un estudio más amplio debería corroborarlo. Se trata de algo perfectamente posible, pues la metodología, en

18 El DETEMA señala la presencia de esta voz en textos de especialidad -no vocabularios o diccionarios- del siglo xv, como el Tratado de cirugía de Guido de Cauliaco, conservado en Madrid, BNE I-196 (alopecia), o el Compendio de cirugía (1481) de Guido Lanfranc de Milán (bocio). Botium / asthma no se documentan, con estas grafías, en el DETEMA, pero sí como asma en textos del prerrenacimiento; tal es el caso del Compendio de la humana salud (1494) y de otras obras contemporáneas. 
todo caso, era común en ambas obras: en Dioscórides se incorporan los términos de otras lenguas en un apartado llamado "Nomina", mientras que en Toxites se dan en listado; la diferencia está en que, al haber suprimido cualquier comentario, Toxites hace patente que su interés se concentra sólo en el léxico.

Estructuralmente, veamos como ejemplo el término came$l l o$, en el que se encuentran tanto similitudes como divergencias en la transcripción de las voces equivalentes en otras lenguas; luego señalaremos otras voces donde la dependencia se muestra con mayor claridad:

TABLA 2

Comparación de voces: Gesner (1541) - Toxites (1574)

Gesner (De Quadrapedibus I, 1551, Toxites

pp. 162-164)

camelus

hebraice gamal

arabice gemal

persice schetor

shymel / zemel

it. \& hisp. camello

gall. chameau

germanice kaemelthier

anglice camel

illyrice vuelblud

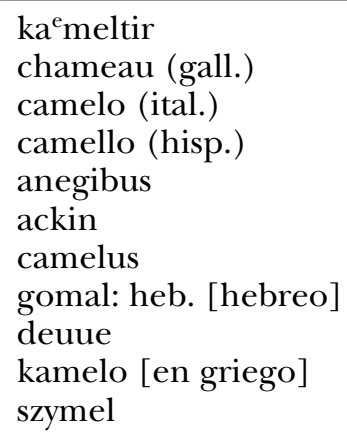

Una fuente que sí fue expresamente usada -pues se cita en el prólogo al lector- es el Nomenclator (1567) de Hadrianus Junius, cuya primera edición, al igual que los comentarios al Dioscórides de Laguna, se publicó en Amberes y contó con innumerables ediciones posteriores (algunas de ellas con añadidos específicos para el público alemán, en cuya región se imprimiría también varias veces entre 1570 y 1577). La nomenclatura de Junius contiene capítulos relativos a voces de plantas, frutos, animales, piedras y medicamentos ${ }^{19}$. Veamos el siguiente

19 Señalamos algunos de estos capítulos: "De animalibus quadrupedibus"; "De avibus"; "De piscium"; "De aromatibus \& speciebus"; "De frumentis \& leguminibus"; "De re herbaria"; "De arboribus \& fruticibus"; "Arborum nomina"; "De metallis"; "De lapidibus"; "De gemmis"; "De medicamentis". 
ejemplo, por medio del cual hemos visto semejanzas entre el capítulo "Frumenta \& legumina" de Toxites y el capítulo "Frumentum" de Junius:

\section{TABLA 3}

Comparación de voces: Junius (1567) - Toxites (1574)

\begin{tabular}{ll}
\hline Junius & Toxites \\
\hline Triticum. Pyrós. Al. Weissen/ & Weizen/Weissen/Terwe. Gall. \\
Weitzen. B. Terwe. G. Fourment. It. & $\begin{array}{l}\text { Froument. Ital. Grano. Hisp. Trigo. } \\
\text { Amest. Chamebe. Dagan. Dagarum. }\end{array}$ \\
& $\begin{array}{l}\text { Frumentum. Heuda. Hyttim. Mest. } \\
\text { Piron. Pyrós. Triticum. }\end{array}$ \\
$\begin{array}{ll}\text { Frumentum, adoreum semen. } \\
\text { Colum. semen cereale [sigue la } \\
\text { equivalencia en griego]. Al. Allerley } \\
\text { korn das atehat. B. koren. G. }\end{array}$ & $\begin{array}{l}\text { Korn. Areforn. Gall. Fourment. } \\
\text { quelquezieredes. Anea. Corotrum. }\end{array}$ \\
$\begin{array}{l}\text { Fourment. It. Ogni sorte di biaua. } \\
\text { H. Los panes quelqueziéredes. }\end{array}$ & Iuminzum. Hanea. Henta. \\
\hline
\end{tabular}

$\mathrm{Al}$ igual que en "los panes quelqueziéredes", Junius parece haber sido indiscutiblemente la fuente de donde Toxites extrajo las equivalencias españolas. Esto es patente por tres motivos: el primero, porque una y otra obra incluyen exactamente los mismos equivalentes; segundo, porque ambas los dan en su mismo orden y, tercero, porque se copian con su misma ortografía -que varía, en ocasiones, de la que usan los demás diccionarios bilingües de los siglos XVI y XVII con el español-, hasta el punto de copiar incluso las erratas.

Así, que Junius fue la fuente de Toxites se demuestra en entradas como la correspondiente al azafrán salvaje, cárcamo alacor o semiente de papagayos: al copiar de Junius se introdujo en Toxites una errata (cártamo > cárcamo) y no se añadió la cedilla esperable en español (alaçor), pues se respetó lo que aparecía en Junius: "Cnicus, hortensis crocus, crocus Sarracenus, offic. certamus. $\chi \vee \tilde{\eta} \mu \circ \zeta$. AL. Wilder Saffran. B. Wild saffraen G. Saffran bastard et sauuage IT. Zaffrano Sarracinesco H. Cártamo, alacor, semiente de papagayos" (entiéndase 'alazor'). El Dioscórides anotado por Laguna fue a su vez fuente de Junius, pero no pudo ser para esta voz la fuente de Toxites, porque allí no aparece el término alaçor: al hablar de cártamo, Laguna sólo señala el término semiente de papagayos ("Llámase este vegetal 
por los Latinos Cnicus, Crocus Sarracenicus, también Carthamus..., por los Italianos Zafarano Sarrasinesco; por los Castellanos Semiente de Papagayos, o de Papagalos, y también Azafrán Romín”, Del cártamo, cap. 189).

Otras voces como culebrilla, rasina, hiniestra/genestra y berillo vuelven a mostrar ese proceso de copia: al señalar la equivalencia española del término asphaltum (Toxites: "Asphaltum bitumen. Gall. Resine qu'on faict du fange: poix du boue. Ital. Resina de fango, pece de limo. Hisp. Rasina de cieno, pez de lodo"), se introduce la variante rasina, presente en Junius ("Resina, rasina; árbol conífera, resinífera..., adonde deriba la rasina o trementina"); en la entrada "Asaphatum. Gall. Feu volage, galle, gratelle. Ital. La volatica. Hisp. Culebrilla, empeine”, se copia a Junius ("impétigo..., culebrilla, empeine"), y del mismo modo en "Aquilegia, nota herba est alias Cantabrica $\mathcal{E} 0$ aquilina. Gall. Ancolye, Aquilegia. / Genestam uel genistam. Gall. Genest. Hisp. Genestra, hiniesta, retama", en Junius como "genista...: H. Genestra, hiniesta, retama". En cuanto a berillo, berilo era la forma ortográfica usual en diccionarios desde Nebrija; Junius, en cambio, señala "beryllus..., H. berillo", como Toxites: "Berillus, speculum christallinum consecratum. Ital \& Hisp. Berillo”.

Los ejemplos anotados indican que Toxites trabajó con obras disponibles en el ámbito centroeuropeo para añadir las equivalencias españolas a los términos. Otras mencionadas en el prólogo, como el Dioscórides (comentado por Valerius Cordus, anotado por Laguna, Mattioli o Ruelio) y Lonizer, probablemente fueron usadas para extraer los términos de las otras lenguas. Así, Dioscórides parece haber sido la fuente directa de voces como mespero, variante de nispero, que aparece en Laguna y en Toxites, pero no en otras obras de la época. Otra voz que da Toxites en el apartado de "aromata \& species", pan de puerco, también aparece en el Dioscórides de Laguna.

Que acudiera a Gesner o a Junius no sorprende, pues sus obras tuvieron mucha difusión (se imprimieron en Amberes y en otros muchos lugares); el manejo de las restantes fuentes ha de verse como el procedimiento habitual en un ámbito académico donde se estaba al tanto de lo publicado por los otros colegas, aunque fueran de otros países, y parte del intercambio de cartas, libros y conocimiento entre ellos.

En este apartado se ha querido mostrar cómo el carácter aglutinador y sistemático de la obra de Toxites debe ser puesto en paralelo con textos de otras disciplinas como la botánica 
y la historia natural, en que la gran cantidad de denominaciones que daban los distintos autores a una misma planta había creado una pluralidad denominativa tal que se trató de paliar con recopilaciones terminológicas acumulativas.

Aunque el latín era la lengua vehicular empleada comúnmente, en cada región las plantas eran más conocidas por sus nombres en vulgar, junto con otros latinizados, empleados normalmente en las boticas. Complementariamente, existían otros nombres populares. La suma de todas estas denominaciones causaba grandes dificultades para determinar a qué planta se estaba refiriendo un estudioso en su obra. Si el término divergía, sólo el dibujo y sus propiedades (usos farmacológicos) podían determinar la equivalencia.

En cierto momento, la gran divergencia entre todos esos nombres se convirtió en una carga. Para mitigarla, se hizo necesario enumerar todos los nombres con que se designaba una planta y establecer cuáles eran sus equivalentes en cada una de las lenguas y regiones, según los libros de los autores principales. Éste sería el paso previo a la sistematización que realizaría Linneo en el siglo XVIII.

Aunque hay precedentes medievales, el esfuerzo más amplio y significativo de agrupar todos aquellos términos lo hizo Caspar Bauhin (1560-1624), quien había estudiado botánica en Padua y, posteriormente, ejercido como médico en París y Basilea. En 1598 editó la Opera omnia de Mattioli, uno de los más importantes comentaristas del Dioscórides del Renacimiento. En aquella edición añadió una lista de sinónimos que sería el germen de su Pinax theatri botanici (1623), en el cual recogió alfabéticamente los nombres y sinónimos con que habían sido designadas seis mil plantas en todas las obras botánicas precedentes de autores antiguos y modernos (unos ciento setenta y cinco títulos). No obstante, esta obra no era más que un "índice" con el que ayudar a la lectura de las obras de Dioscórides, Plinio y de cualquier otro botánico.

Christian Mentzel (1622-1701) fue un paso más allá. Su formación como lingüista -fue estudioso de la lengua china, sobre la que compuso varias obras, entre ellas un diccionario sino-latinole permitió trasladar sus conocimientos de esta área a otras que también cultivó: la medicina y, sobre todo, la botánica. Su Index nominum plantarum multilinguis (también titulado Pinax botanonymos polyglottos katholikos, 1682) ofrece los nombres latinos de plantas con sus equivalencias en más de cuarenta lenguas (europeas, 
asiáticas, africanas), además de las clásicas (griego, latín, hebreo, caldeo, más árabe). Lo interesante de su Index es que señala entre paréntesis el término que usó cada autor para nombrar una planta y que incluye las voces contenidas en la obra de Bauhin.

La elaboración de estas obras supuso la culminación de un período de gran actividad botánica, en el que hubo de sumarse, junto con las regionales, las floras procedentes de muchas partes del globo. La acumulación denominativa que en ellas se dio permitió la mejora de las clasificaciones posteriores.

\section{OBRAS RELAGIONADAS}

Una vez hecha esta revisión, queremos retomar, para cerrar el análisis, lo dicho sobre el paracelsismo en el primer apartado y asociar la obra de Toxites con otras. Onomastica es sólo uno de los muchos títulos que se imprimieron relacionados con Paracelso, y no el único léxico. Hubo también otros vocabularios relativos a la alquimia basados en sus textos, como el Dictionarium Theophrasti Paracelsi continens obscuriorum vocabulorum quibus suis scriptis passim utitur, definitiones de Gerardo Dorneo (Francfort, 1584), diccionario de las voces de Teofrasto con definiciones en lengua latina, el cual parece seguir en ocasiones al mismo Toxites. Veamos un ejemplo:

Toxites 1574: Aqua lubricata, id est muscilaginosa, ut sunt confectae aquae cum saccharo, iulepi, multaque similia. Alaberige Wasser was mit zucker... Gall. Aigue lubricq, comme sont les boissons faictes de sucre, ou aultres choses.

Dorneo 1584: Aqua lubricata est ea quae fit cum muscilaginosis, ut sunt saccara, iulep \& similia.

Toxites pudo, así, no sólo usar las obras precedentes, sino también repercutir en posteriores.

El interés proseguiría en el siglo XVII, según atestiguan títulos como el Lexicon medico-Galeno, chymico-pharmaceuticum, de Friedrich Müller von Löwenstein (Francfort, 1661), o el Lexicon Chymicum, cum obscuriorum verborum et rerum hermeticarum, tum phrasium Paracelsicarum, in scriptus eijus: et aliorum chymirocrum, passim occurentium, planam explicationem continens, per Gulielmum Johnsonum Chymicum (Londres, 1652), que fue ree- 
ditado en 1660 con un suplemento titulado Lexicon chymicum, continens vocabula chymica in priore libro omissa, multis vocabulorum chymicorum characteribus adjectis è Basilio Valentino, Theophrasto Paracelso, Oswaldo Crollio, aliisque Authoribus Chymicis collectis.

\section{Conclusiones}

En este trabajo nos hemos acercado a la vida de Michael Toxites y a su obra Onomastica; hemos señalado la intención con que fue realizada y citado otros títulos similares que testimonian que la obra Onomastica debe analizarse en la órbita de las obras sobre Paracelso y encuadrarse, desde un punto de vista filológico, con otros vocabularios de especialidad de su época, de medicina, botánica e historia natural.

Considerando la bibliografía disponible, en que la obra de Toxites es ciertamente conocida en su conjunto y puesta en relación con la difusión del paracelsismo, pero no analizada individualmente ${ }^{20}$, el estudio presentado en este artículo es el más detallado hasta el momento tocante a la obra Onomastica. Es además el primero realizado desde una perspectiva lexicológica y el primero que presenta un análisis de las voces españolas en ella incluidas, con ejemplos concretos sobre sus posibles fuentes y su interés para la datación de ciertos términos españoles.

Hemos visto por este análisis y por la estructura de la obra que la labor de Toxites fue acumulativa y que se basó en textos anteriores. Su grado de innovación no es por ello determinante para la lengua española, pero, en cualquier caso, puso en conjunto un gran número de términos que no se incluyó usualmente en los diccionarios de lengua o no lo haría hasta más tarde. Al señalar además equivalencias plurilingües referidas a nombres de plantas, y desgajarlas fuera de cualquier texto explicativo, la obra de Toxites puede considerarse un eslabón temprano de la sistematización terminológica que se llevará a cabo un siglo más tarde por Mentzel o Bauhin en ámbitos como la botánica.

El estudio lexicológico de algunas de las voces muestra que los vocabularios de Toxites complementan las documentaciones ofrecidas por otros títulos contemporáneos en lo relativo a

${ }^{20}$ Sólo Soukup (1997, pp. 224-235) se detiene en ella, pero únicamente transcribe algunas calas de la obra con los términos en alemán para comentarlos a partir de su relación con el paracelsismo. 
la datación de voces. Resaltamos por ello, desde aquí, la necesidad de detenerse en este tipo de estudios filológicos de las obras técnicas del Renacimiento y Barroco para conocer cómo se enriquecieron las lenguas vulgares -ya sea la española, la francesa o la inglesa- en un momento crucial de su desarrollo. Aunque hubiera estudios derivados de una perspectiva de la historia de la ciencia o de las ideas, hemos de preocuparnos también de analizar las obras desde una perspectiva filológica, pues es tarea que no harán investigadores de otras disciplinas; sólo así podremos ayudar a establecer qué procedimientos neológicos se realizaron, cómo se fueron incorporando los cultismos griegos y latinos en los ámbitos de especialidad y qué préstamos se dieron entre unas lenguas y otras, lo que redundará en beneficio de la historia de nuestras respectivas lenguas.

\section{REFERENCIAS}

$C D H=$ Instituto de Investigación Rafael Lapesa de la Real Academia Española 2013. Corpus del Nuevo diccionario histórico del español [en línea], http:/ / web.frl.es/CNDHE [consultado el 22 de abril de 2018].

CORDE $=$ Real Academia Española: Banco de datos [en línea]. Corpus diacrónico del español, http://www.rae.es [consultado el 28 de febrero de 2017].

DAniel, Dane T. 2007. "Coping with heresy: Suchten, Toxites, and the early reception of Paracelsus's theology", en Chymists and chymistry: Studies in the history of alchemy and Early Modern chemistry. Ed. Lawrence Principe, Watson Publishing-Chemical Heritage Foundation and Science History, Sagamore Beach, MA, pp. 53-62.

DETEMA = María Teresa Herrera (dir.) 1996. Diccionario español de textos médicos antiguos, Arco/Libros, Madrid, 2 vols.

Dioscórides, PEDACio 1555. Acerca de la materia medicinal y de los venenos mortíferos traduzido de lengua griega en la vulgar castellana $\mathcal{E}$ illustrado con claras y substantiales annotationes... por Andrés de Laguna, Iuan Latio, Anuers.

DLE = Diccionario de la lengua española 2014. 23a ed., RAE-Espasa, Madrid.

Dorneo, G. 1584. Dictionarium Theophrasti Paracelsim continens obscuriorum vocabulorum, quibus suis scriptis passim utitur, definitiones, Francoforti.

Ferguson, John 1877-1896. Bibliographia Paracelsica. First series, parts I-VI, printed at the University Press by Robert Maclehose, Glasgow.

GESNER, KonRAD 1551-1558. Historiae animalium Liber I de quadrupedibus uiuiparis. Tiguri, apud Christ. Froschoverum; Historiae animalium Liber II de quadrupedibus ouiparis, 1554, vol. 2; Historiae animalium Liber III, qui est de auium natura, 1555, vol. 3; Historiae animalium Liber IIII qui est de piscium $\mathcal{E}$ aquatilium animantium natura, 1558, vol. 4; Historia animalium Lib. V, qui est de serpentium natura, vol. 5. [He consultado el ejemplar de una edición de 1587]. 
Gilly, Carlos 2010-13. “Zwinger e Paracelso”, Azogue, 7, pp. 298-307, http:/ / www.revistaazogue.com/Azogue7-8.pdf.

GutiÉRrez Rodilla, Bertha 2007. La esforzada reelaboración del saber: repertorios médicos de interés lexicográfico anteriores a la imprenta, Cilengua-Instituto Historia de la Lengua, San Millán de la Cogolla. (Monografías, 2).

Herrera, María Teresa (dir.) 1996. Diccionario español de textos médicos antiguos, Arco/Libros, Madrid.

JunIUs, H. 1567. Nomenclator omnium rerum propria nomina variis linguis explicata indicans, Christophorus Plantinus, Antuerpiae.

Kühlmann, Wilhelm \& Joachim Telle (eds.) 2004. Corpus Paracelsisticum: Dokumente frühneuzeitlicher Naturphilosophie in Deutschland = Documents of Early Modern Age nature philosophy in Germany. Vol. II: Early paracelsianism: Part two, DeGruyter, Berlin. (Frühe Neuzeit, 89).

Nieto Jiménez, Lidio y Manuel Alvar EzQuerra 2007. Nuevo tesoro lexicográfico del español (s. XIV-1726), Arco/Libros, Madrid, 11 vols.

$N T L L E=$ Real Academia española [en línea]. Nuevo tesoro lexicográfico de la lengua española, http://ntlle.rae.es/ntlle/SrvltGUILoginNtlle [consultado el 22 de abril de 2018].

Pablo Núñez, Luis 2010. El arte de las palabras: diccionarios e imprenta en el Siglo de Oro, Editora Regional de Extremadura, Mérida.

Pablo Núñez, Luis 2012. Hacia una flora universal. La Botánica y el español como lengua de la ciencia, Cilengua-Fundación San Millán, San Millán de la Cogolla. (Monografías del Instituto Historia de la Lengua, 11).

Pantin, Isabelle 2010. "El papel de las traducciones en los intercambios científicos europeos de los siglos xvi y xviı", en La traducción cultural en la Europa Moderna. Eds. Peter Burke y R. Po-Chia Hsia, Akal, Madrid, pp. 195-215.

Rodríguez Guerrero, José 2001. "Censura y paracelsismo durante el reinado de Felipe II”, Azogue, 4, http:// www.revistaazogue.com/ inquisicion.htm.

Sarcilly, Charles 1950 [1631]. L'art d'alchimie et autres écrits de Théoph. Paracelse Bombast...: tirés des traductions de ses premiers sectateurs français... auxquels on a ajouté en Appendice les Opinions du Docteur Toxites Ẽ Jean Wier, Presses Littéraires de France, Paris. [Ed. facs. de XIV Livres des paragraphes, Hervé du Mesnil, Paris].

Schmidt, Charles 1888. Michael Schütz, genannt Toxites: Leben eines Humanisten und Arztes aus dem 16. Jahrhundert, F. Bull, Strassburg.

Sommerhalder, Hugo 2018. Johann Fischarts Werk, De Gruyter, Berlin-Boston.

SOUkup, R. Werner 2007. Chemie in Österreich: von den Anfängen bis zum Ende des 18 Jahrhunderts, Böhlau, Wien.

Soukup, R. Werner und Helmut Mayer 1997. Alchemistisches Gold, Paracelsistische Pharmaka: Laboratoriumstechnik im 16. Jahrhundert, Böhlau, Wien.

SudHoff, KARL 1894. "Ein Beitrag zur Bibliographie der Paracelsisten im 16. Jahrhundert”, Centralblatt für Bibliothekswesen, 10, pp. 316-326 y 385-407.

SudHOFf, KARL 1958 [1894]. Bibliographia Paracelsica: besprechung der unter Hohenheims Namen 1527-1893 erschienen Druckschriften, Akademische Druck-U. Verlagsanstalt, Graz. 
Tonelli, Giorgio 2006. A short-title list of subject dictionaries of the sixteenth, seventeenth and eighteenth centuries. Ed. revised and annotated by Eugenio Canone \& Margherita Palumbo, Leo S. Olschki, Firenze.

Toxites, Michael 1567. Spongia Stibii, adversus Lucae Stenglini medicinae doctoris et physii Augustani aspergines. Autore Micaelo Toxite Rhaeto, medico argentoratensi: Theophrasti Paracelsi discipulo, Strassburg.

Toxites, Michael 1574. Onomastica II. I Philosophicum, medicum, synonymum ex varijs vulgaribusque linguis. II Theophrasti Paracelsi: hoc est, earum vocum, quarum in scriptis eius solet usus esse, explicatio. Nunc primum in commodum omnium philosophiae, ac medicinae Theophrasticae studiosorum, cuiuscunque nationis sint: fideliter publicata, Bernhardum Iobinum, Argentorati. 